\title{
Los nuevos proyectos de integración regional vigentes en América Latina: la Alternativa Bolivariana para Nuestra América y la Comunidad Sudamericana de Naciones
}

\section{Carlos Nahuel Oddone ${ }^{1}$ y Leonardo Granato ${ }^{2}$}

\section{RESUMEN}

El presente trabajo se centra en abordar el origen y los principios y líneas de acción de dos nuevas propuestas de integración regional en América Latina: la Alternativa Bolivariana para Nuestra América (ALBA) y la Comunidad Sudamericana de Naciones (CSN). En este sentido, y en aras de vislumbrar el movimiento que le den a ambos proyectos los Estados que los integran; se comenta brevemente la postura de los diferentes países de la región frente a los mismos. Asimismo, en virtud de entender la autonomía como fin último del ALBA y CSN, es que se encuadrará a los mismos dentro de dos tipos específicos de autonomía: la autonomía secesionista y la autonomía relacional.

\section{Palabras clave:}

América Latina - Alternativa Bolivariana para Nuestra América - Comunidad Sudamericana de Naciones - Autonomía - Integración Regional.

\section{RESUMO}

O presente artigo aborda a origem e os princípios e linhas de ação de duas novas propostas de integração regional vigentes na América Latina: a Alternativa Bolivariana para Nossa América (ALBA) e a Comunidade Sul-Americana de Nações (CASA). Nesse sentido, e em prol de vislumbrar o movimento que lhe outorguem a ambos projetos os Estados que os

\footnotetext{
${ }^{1}$ Licenciado en Relaciones Internacionales por la Universidad Nacional del Centro de la Provincia de Buenos Aires, Argentina. Master en Derecho de la Integración Económica de la Universidad del Salvador, Argentina; en convenio con l'Université Paris 1 Panthéon-Sorbonne, Francia. Candidato al Master en Integración Económica Global y Regional de la Universidad Internacional de Andalucía, España. Contacto: oddone.nahuel@gmail.com

${ }^{2}$ Abogado por la Universidad de Belgrano, Argentina. Master en Derecho de la Integración Económica de la Universidad del Salvador, Argentina; en convenio con l'Université Paris 1 Panthéon-Sorbonne, Francia. Contacto: granato.leonardo@gmail.com
} 
integram, comenta-se brevemente a postura dos diferentes países da região diante dos mesmos. Todavia, em virtude de entender a autonomia como fim último da ALBA e CASA, é que ambos serão enquadrados dentro de dois tipos específicos de autonomia: a autonomia da secessão y a autonomia relacional.

\title{
Palavras-chave:
}

América Latina - Alternativa Bolivariana para Nossa América - Comunidade SulAmericana de Nações - Autonomia - Integração Regional.

\begin{abstract}
The present work is focused on approaching the origin, the principles and the course of action of two new proposals of regional integration in Latin America: the Bolivarian Alternative for the Americas (BAA) and the Southern Community of Nations (SCN). On our humble point of view, we identify a kind of specific autonomy in each process of integration. We aim at proving that the BAA would suit the "secessionist autonomy" whereas the latter project, the SCN, could suit the "relational autonomy".
\end{abstract}

\section{Key words:}

Latin America - Bolivarian Alternative for the Americas - Southern Community of Nations - Autonomy - Regional Integration.

\section{INTRODUCCIÓN}

En los últimos años, han surgido en América Latina dos proyectos de integración regional; el primero de ellos, la Alternativa Bolivariana para Nuestra América (ALBA), impulsado por la República Bolivariana de Venezuela y cuyo origen se encuentra en la oposición de éste país a las negociaciones del Acuerdo de Libre Comercio para las Américas (ALCA). El segundo proyecto es el de la Comunidad Sudamericana de Naciones (CSN), cuya génesis está vinculada a propiciar una nueva visión del escenario sudamericano.

Ambas propuestas parecen responder al leit motiv del desarrollo de los países de la región. Sin lugar a dudas, sus respectivos ejes de acción abordan temáticas / problemáticas; 
que necesariamente deben ser reconocidas, y resueltas en caso de éstas últimas, por los Estados en su conjunto.

Las crisis económico-institucionales que durante las últimas décadas han atravesado los países de la región, desde la crisis de la deuda de los años ochenta hasta las crisis económico-financieras de los años noventa pasando por innumerables crisis de corrupción y gobernabilidad, ponen de manifiesto que nuestros países deben recuperar la senda del desarrollo.

El inicio del siglo XXI ha encontrado a los países sudamericanos con el regreso de los liderazgos carismáticos y una tendencia de la izquierda a hacerse con el poder; es en este contexto que la integración vuelve a convertirse en un elemento aglutinador de los deseos autonómicos sudamericanos, presentándose como alternativa heterodoxa a las propuestas de integración neoliberal vía tratados de libre comercio (en adelante TLC) propugnadas por los Estados Unidos como potencia hegemónica del sistema.

Se parte de la premisa que en un mundo globalizado los mejores resultados se logran sobre bases integradas, y ello se ve reflejado en los puntos de partida de ambos proyectos de integración.

\section{DESCRIPCIÓN DEL TRABAJO}

La metodología de exposición del presente trabajo responde a una variable cronológica, por lo que en primer se tratará la propuesta ALBA surgida en 2001, y en segundo lugar, la de la CSN de 2004.

El análisis de ambos programas de integración se centra en abordar sus respectivos orígenes, estudiar sus principios y líneas de acción, comentar las posturas de los países de la región frente a éstos y por último, encuadrarlos dentro de dos tipos específicos de Autonomía: la Secesionista y la Relacional.

El presente trabajo es un estudio de las propuestas de integración regional de ALBA y CSN identificadas en su faz internacional con dos modelos de autonomía. 


\section{LA ALTERNATIVA BOLIVARIANA PARA NUESTRA AMÉRICA (ALBA)}

\section{GÉNESIS DEL ALBA}

Las negociaciones en torno al ALCA tuvieron su inicio en la Cumbre de las Américas celebrada en la ciudad de Miami en diciembre de 1994 y se extenderían a lo largo del resto de la década de los años noventa y el primer lustro del siglo $\mathrm{XXI}^{3}$.

La firma de ese tratado hubiera supuesto la creación de un área de libre comercio que abarcaría todo el continente americano inspirada en los principios de preservar y fortalecer la democracia representativa, promover la prosperidad a través de la creciente integración económica y el libre comercio entre los países del hemisferio, eliminar progresivamente las barreras al comercio de bienes y servicios y la inversión, maximizar la apertura de los mercados mediante altos niveles de disciplina a través de un acuerdo balanceado y comprensivo, proporcionar oportunidades para facilitar la integración de las economías más pequeñas, procurar que las políticas ambientales y de liberalización comercial se apoyen mutuamente, y asegurar de conformidad con las respectivas leyes y reglamentos, la observancia y promoción de los derechos laborales renovando el compromiso de respetar las normas fundamentales del trabajo internacionalmente reconocidas.

Según Montero Soler (2007: 3) "la esencia de ese proyecto era imponer, por la vía aparentemente neutral del libre comercio, la progresiva desarticulación de los pocos ámbitos económicos y sociales sobre los que los Estados seguían manteniendo, tras la ofensiva neoliberal, un cierto control".

Producido el estancamiento de las negociaciones conjuntas, los Estados Unidos abandonaron su proyecto original y optaron por su continuidad agrupando a los interlocutores que estuvieran dispuestos a suscribir el acuerdo por grupos regionales. Se negoció y firmó un TLC con Chile, otro con Centroamérica y la República Dominicana (CAFTA) y se iniciaron las negociaciones con Colombia, Perú y Ecuador de la Comunidad

\footnotetext{
${ }^{3}$ El proyecto ALCA surge de la Iniciativa para las Américas (IPA) presentada por el presidente de los Estados Unidos George Bush en junio de 1990, siendo reactivado por su sucesor William Clinton en la Cumbre de Miami ya citada. La IPA constituyó la primera declaración de política económica de los Estados Unidos hacia América Latina desde la Alianza para el Progreso (1962) de John F. Kennedy, y al igual que ésta última propuesta, la IPA tuvo una reacción favorable en la subregión. No obstante, esta propuesta no fue concretada en ningún instrumento específico (Oddone y Granato, 2006: 48).
} 
Andina de Naciones (CAN), sobre una base de múltiples acuerdos bilaterales de negociación.

Es en este contexto antes descrito que en diciembre del año $2001^{4}$ surge la propuesta venezolana de integración Alternativa Bolivariana para Nuestra América (ALBA), y al decir de Montero Soler (2007: 5) "el surgimiento de la propuesta (...) se está configurando como proyecto contrahegemónico en el contexto de los vertiginosos cambios políticos, económicos y sociales que experimenta América Latina desde los últimos años del siglo $\mathrm{XX}{ }^{\prime 5}$.

Desde el propio surgimiento el ALBA se entendió como una alternativa que constituye un opuesto al ALCA. En este sentido, es difícil pensar el surgimiento del ALBA sin el antecedente del ALCA.

Continua diciendo el autor antes mencionado: "Esta propuesta marca un distanciamiento, no sólo del ALCA, sino también de los proyectos de integración que le han precedido y que parte, de entrada, de su naturaleza eminentemente antiimperialista" (Montero Soler, 2007: 5) ${ }^{6}$.

Como sostuvo el propio presidente Chávez: “decía que tenemos una doble tarea, enterrar el ALCA y el modelo económico, imperialista, capitalista por una parte, pero por la otra a nosotros nos toca, (...), ser los parteros del nuevo tiempo, los parteros de la nueva historia, los parteros de la nueva integración, los parteros del ALBA"

\section{PRINCIPIOS DEL ALBA}

La pobreza de la mayoría de la población, las profundas asimetrías entre países, el intercambio desigual, el peso de una deuda significativa, la imposición de las políticas de ajuste estructural del Fondo Monetario Internacional (FMI) y del Banco Mundial (BM), el desigual acceso a la información y al conocimiento y los problemas que afectan la

\footnotetext{
${ }^{4}$ Durante la III Cumbre de Jefes de Estado de la Asociación de Estados del Caribe de diciembre de 2001.

${ }^{5}$ Sin embargo, había sido el propio presidente venezolano Rafael Caldera quien suscribiera en octubre de 1997 siete instrumentos con los Estados Unidos que tenían como objetivo común la puesta en marcha del ALCA para 2005.

${ }^{6}$ Según Katz (2006) el ALBA carece de una definición perfectamente delimitada y que pudiera ser asimilable a la de otros procesos de integración regional.

${ }^{7}$ Discurso en la Clausura de la III Cumbre de los Pueblos de América. Estado Mundialista de Mar del Plata, Argentina; 4 de noviembre de 2005. Accesible desde: http://www.alternativabolivariana.org/pdf/ discurso Chavez MardelPlata.pdf
} 
consolidación de verdaderas democracias son importantes obstáculos para esta integración latinoamericana.

El ALBA parece haber optado por "la lógica de la cooperación comercial, el intercambio solidario y la complementariedad económica entre las diferentes estructuras productivas nacionales como principios generales de su filosofía” (Montero Soler, 2007: 5).

En este sentido, la Alternativa está conformada por una serie de acuerdos cuyos objetivos desembocan en todo momento en la búsqueda de la superación de las asimetrías económicas y sociales existentes en los países latinoamericanos ${ }^{8}$.

Es por ello que parecería haberse afirmado que el ALBA promueve un régimen de ventajas cooperativas, en el sentido que "cada país pone sus ventajas comparativas para la producción de determinadas bienes y servicios al servicio de la complementariedad entre las diferentes economías nacionales a partir de un esquema de cooperación basado, (...) en el intercambio solidario y en la existencia de intereses y necesidades mutuas" (Montero Soler, 2007: 6).

Se entiende que este esquema de intercambio comercial lograría por un lado, profundizar en una especialización productiva eficiente y competitiva, compatible con un desarrollo económico y social equilibrado al interior de cada Estado; y por otro, permitir el distanciamiento de las estructuras productivas nacionales de las imposiciones y condicionantes del mercado mundial globalizado.

Como sostiene Martínez (2004) el ALBA es una estrategia glocal desde la necesidad de afrontar económica, ideológica, política y culturalmente; la legitimización institucional del mercado mundial.

El fenómeno de la glocalización sólo en parte tiene una caracterización de tipo económica, destacándose por sobre todo el componente politológico y sociocultural que definirían una nueva relación entre individuo y contrato, entre comunidad e instituciones, entre local government y global government. Como ha sostenido Boisier (2005: 50): "Entre la alienación total y la marginación completa surgen el sincretismo y la cultura 'híbrida' de García Canclini. Al contrario de lo que sostiene Bauman, ser local en un mundo globalizado no es una señal de penuria y degradación social. La síntesis se encuentra más bien en el neologismo de Robertson, el término 'glocal'".

\footnotetext{
${ }^{8}$ Una recopilación de los convenios suscriptos en este marco puede ser encontrada en el sitio oficial del
} ALBA en Internet, accesible desde http://www.alternativabolivariana.org 
Para Martínez (2005: 2): "El ALBA, considerado como una estrategia glocal, propia de un proyecto integrador latinoamericano, y desde una interpretación economicista como una estrategia periférica, permitiría la redistribución de las riquezas naturales y manufacturadas en los contextos neoculturales que luchan por su propia autodeterminación, y desde donde el intercambio social, cultural y económico sea realmente desde un interés humano por lo humano, posee dentro uno de sus puntos más importantes: el desarrollo desde las propias dinámicas culturales, esto es un desarrollo endógeno contrario incluso al desarrollo autogestionado proclamado e instaurado por el Banco Mundial en los países en vías de desarrollo".

\section{INSTRUMENTOS Y PLAN ESTRATÉGICO DEL ALBA}

Tal como se mencionó el ALBA toma distancia de los esquemas de integración de corte netamente económico para situar la llamada "deuda social" en el centro de su estrategia integracionista. Reflejo de ello son los preceptos contenidos en los convenios suscriptos por los dos Estados promotores de la Alternativa que colocan, el desarrollo de los servicios públicos venezolanos (con especial énfasis en salud y educación) y el abastecimiento energético cubano; en el centro de su agenda comercial bilateral.

En este contexto, y en virtud de la cooperación cubana; Venezuela ha instrumentado las denominadas "Misiones" obteniendo óptimos resultados en materia de alfabetización, escolarización y asistencia sanitaria básica de las clases populares. Como contrapartida, Venezuela ha ofrecido a la República Cubana un trato preferencial en la venta de petróleo según Convenio Integral de Cooperación entre ambos países suscripto el 30 de octubre de 2000.

Se debe destacar también la cooperación en materia de ciencia y tecnología, cuidado medioambiental y académico - profesional, en especial en el ámbito de la medicina. En virtud del Acuerdo para la aplicación del ALBA y el Tratado de Comercio de los Pueblos firmado en La Habana el 29 de abril de 2006, la República de Bolivia se incorpora a esta relación de cooperación de Cuba y la República Bolivariana de Venezuela 9 .

\footnotetext{
${ }^{9}$ La incorporación de un país pobre como Bolivia al proyecto integracionista en abril de 2006 tras la llegada a la presidencia de Evo Morales, constituye claramente uno de los principales avances en la propagación del ALBA en América Latina y en la contribución al desarrollo social y económico del Estado, dado que tanto Venezuela como Cuba se han comprometido a contribuir en la implementación de programas sociales en
} 
No se puede dejar de mencionar el Acuerdo de Cooperación Energética PETROCARIBE suscripto en Puerto la Cruz el 29 de junio de 2005 por Antigua y Barbuda, Bahamas, Belice, Cuba, Dominica, República Dominicana, Granada, Guyana, Jamaica, San Vicente y las Granadinas, Santa Lucía, San Cristóbal y Nieves, Surinam y Venezuela. Para contribuir con el desarrollo económico y social de los países del Caribe, PETROCARIBE dispondrá de un Fondo destinado al financiamiento de programas sociales y económicos, con aportes provenientes de instrumentos financieros y no financieros; contribuciones que se puedan acordar de la porción financiada de la factura petrolera y los ahorros producidos por el comercio directo. Este Fondo se denominará ALBA-CARIBE. Con el propósito de activar el Fondo ALBA-CARIBE, la República Bolivariana de Venezuela aportará un capital inicial de cincuenta millones de dólares (US\$ 50.000.000,oo).

En el plan estratégico del ALBA también se contemplan cuestiones que hacen al intercambio comercial estrictu sensu: un trato preferente para los inversores nacionales de uno u otro Estado en los respectivos territorios nacionales; utilización de mecanismos de comercio compensado; utilización de las respectivas monedas nacionales para el pago de las transacciones comerciales entre los países; eliminación de barreras para arancelarias a las importaciones de productos entre los Estados; eliminación de la imposición sobre utilidades de las inversiones estatales, mixtas e incluso privadas en tanto produzcan el recupero de la inversión; trato preferente al transporte marítimo y aéreo en territorio nacional de las compañías de la subregión y financiación de proyectos productivos en sectores estratégicos para las economías.

Sin lugar a duda la cooperación en materia de energía se erige como uno de los principales ejes de la integración ALBA para así intentar reducir las asimetrías regionales a través de la amortiguación de los fuertes impactos que el alza continuada de los precios del petróleo durante los últimos años ha supuesto para las frágiles economías de la zona.

En este sentido, como sostiene Montero Soler (2007:10): "Venezuela percibe así, que, en unos tiempos en los que la lucha por los recursos naturales determina en gran medida la geopolítica mundial, la integración energética puede ser el mecanismo más incisivo para conseguir avanzar hacia una auténtica comunidad de naciones; comunidad a la

materia sanitaria y educativa. 
que, por otra parte, aspira a contagiar de los valores que informen el proceso de transformación social que está realizando en su territorio".

\section{AVANCES EN LA INTEGRACIÓN ALBA}

Sin perjuicio de las relaciones estrechadas por Venezuela y Cuba, el ALBA ha ido extendiendo su presencia en el resto del continente, ya sea a través de acuerdos ${ }^{10} \mathrm{o}$ bien, a través de la primera cadena de televisión pública latinoamericana: TeleSur.

Pero sin lugar a dudas, la incorporación de Venezuela al MERCOSUR a través del Protocolo de Adhesión de fecha 4 de julio de 2006 abre un nuevo camino para la expansión de este proyecto integrador de América Latina. Ejemplo de ello fue el discurso del presidente Chávez en la XXX Cumbre Presidencial del MERCOSUR de julio de 2006 que se estructuró en torno a la idea que "la integración social es el único camino que puede hacer viable la soberanía de los países de América Latina frente al proyecto imperialista estadounidense".

En este sentido, el presidente Chávez ha propuesto la creación del denominado "Bono del Sur", destinado a financiar proyectos productivos en las zonas más atrasadas; y el "Banco del Sur", cuya misión sea la de financiar significativos proyectos de infraestructura y desarrollo social en toda Latinoamérica; que se sumarían a las propuestas en materia de energía e infraestructura, concebidos éstos como elementos autonomizantes del Cono Sur.

Desde una perspectiva geopolítica, la incorporación de Venezuela al MERCOSUR puede interpretarse como un juego estratégico en el cual Brasil -socio mayor del MERCOSUR- acepta la aspiración de Argentina de contrapesar su peso específico en una alianza con el país bolivariano. Todo esto redundará en un nuevo MERCOSUR ampliado que supere lo económico - comercial, para avanzar sobre lo social y lo político, y en la constitución de un nuevo espacio en donde las propuestas ALBA y MERCOSUR puedan confluir desde la sectorialidad del primero ${ }^{11}$ y la generalidad del segundo.

\footnotetext{
${ }^{10}$ Dentro de los acuerdos ALBA, propiamente dichos, se encuentran instrumentos suscriptos entre Venezuela y Cuba por un lado, y los países del Caribe por otro. Asimismo lo han hecho con Bolivia. En materia bilateral, Venezuela ha emitido comunicados conjuntos con Argentina y Brasil y ha firmado convenios con Bolivia y Uruguay.

11 “La geometría variable del ALBA se manifiesta en acuerdos puntuales, como la creación de TeleSur, el Banco del Sur, el Bono del Sur, eventualmente la Universidad del Sur, y en una serie de transacciones
} 


\section{LOS PAÍSES DE LA REGIÓN FRENTE AL ALBA}

Para Cuba la propuesta ALBA se presenta como la oportunidad de "una verdadera integración". Se podría sostener que éste país ha sido el más alejado de la integración en América Latina debido a que por su régimen de gobierno no ha sido invitado a formar parte de numerosas propuestas, sean éstas de origen propiamente latinoamericano o evidentemente norteamericanas, en las cuales la presencia de la República Cubana es impensable. Nunca ha llegado a pensarse un ALCA con Cuba.

De todas formas, en pos de la incorporación de Cuba a los sistemas de integración subregionales se destaca la adhesión de Cuba a la Asociación Latinoamericana de Integración (ALADI) desde noviembre de 1998, fecha a partir de la cual se observa un doble proceso: por un lado, una mayor apertura de Cuba hacia el mundo; y por otro lado, una "nueva y renovada consideración latinoamericana" de Cuba.

En el caso argentino el apoyo a la propuesta ALBA se entiende en el marco del afianzamiento de la relación bilateral con Venezuela, el aumento de inversiones, la creación de los ya mencionados Bono y Banco del Sur que pretenden dar estabilidad al peso argentino y favorecer la creación de una moneda común basada en los precios del crudo venezolano y, la complementación energética. En este marco no se puede dejar de mencionar que Venezuela es unos de los países que más bonos ha comprado de la deuda argentina por un valor nominal de tres mil doscientos cuarenta millones de dólares. Asimismo, los intereses de Argentina se centran en una posible asociación entre la empresa Energía Argentina S. A. (ENARSA) y la empresa Petróleos de Venezuela S. A. (PDVSA), a lo que se sumaría la construcción del gasoducto del sur en los territorios de Venezuela, Brasil y Argentina.

concretas, como el aporte de recursos venezolanos para impedir la extranjerización de la cooperativa Sancor, a cambio de entregas de leche en polvo y transferencia de tecnología alimentaria. En la misma lógica se inscriben el intercambio de fuel oil por bovinos; el tratado energético supranacional de la Organización de Países Productores y Exportadores de Gas (OPEGASUR); la participación de PDVSA en el gasoducto del Nordeste; las cartas de intención firmadas para que empresas argentinas siembren soja, girasol y algodón en un centenar de miles de hectáreas que PDVSA posee en su país; para la instalación en Venezuela de un centro genético de semen de vacunos de la raza Brangus o la venta llave en mano de laboratorios argentinos de investigación sobre la papa o la genética animal; la producción de partes y el armado de autobuses a gas argentinos en Venezuela o la participación de ENARSA en la explotación de petróleo pesado en la franja del Orinoco" (Verbitsky, 2007). 
Por su parte, en el caso brasileño muchos creen en una eventual alianza empresaria entre PDVSA y Petróleo Brasileiro S. A. (PETROBRAS); la construcción de infraestructura física conjunta ${ }^{12}$ y el gasoducto del sur que atravesará todo el territorio brasileño en su amplitud. Por lo general, la cautela en política exterior ha sido la posición que podría caracterizar al vecino país de la República Federativa de Brasil.

En el caso de la República de Bolivia, más allá de la identificación ideológica entre el presidente Evo Morales y el presidente venezolano Chávez; amplio interés ha despertado en éste primer país la futura concreción del Banco del Sur pues le permitiría satisfacer sus necesidades financieras y respaldar su moneda vis-à-vis aumenta los márgenes de autonomía frente a los Estados Unidos y los inversores extranjeros presentes en el mismo. En este contexto, Bolivia ha calificado al ALBA como "una propuesta verdaderamente solidaria".

Antigua y Barbuda, Dominica y San Vicente y las Granadinas son los países de la Comunidad Caribeña (CARICOM) que participaron en la firma del Memorando de Entendimiento con Venezuela. Para los países caribeños acercarse al ALBA implica poder tomar una mínima distancia de la presencia de Estados Unidos en la subregión, a la cual siempre ha considerado "su patrio trasero".

Por su parte, para Jamaica, el amplio interés suscitado por la propuesta ALBA se debe básicamente por una cuestión energética, fiel reflejo de ello es el entendimiento suscripto por éste país y Venezuela para la conformación de un Comité de Dirección de Alto Nivel para el estudio de la expansión de la infraestructura del gas; proyecto que desarrollarán conjuntamente PDVSA y la Petrolium Corporation of Jamaica.

Colombia puede considerarse el país que mayor indiferencia ha demostrado frente a la propuesta ALBA. La alineación cuasi automática de la Presidencia de Álvaro Uribe con los Estados Unidos no permite un apoyo al ALBA, lo que implicaría una contradicción con su actual política exterior de "respice polum". En este sentido, los temas de agenda de la relación bilateral continúan siendo la ratificación del TLC, la incorporación de Colombia al Tratado sobre Biocombustibles que ha firmado los Estados Unidos con el Brasil y la continuación del Plan Colombia. Asimismo, en Costa Rica y Guatemala se pueden observar posiciones similares a las de Colombia.

\footnotetext{
${ }^{12}$ Ya se han iniciado obras de carreteras entre el sur de Venezuela y el norte de Brasil.
} 


\section{LA AUTONOMÍA SECESIONISTA DE LA PROPUESTA ALBA}

Siendo el grado de autonomía adquirido o a adquirir un dato clave al momento de analizar la política exterior de un país o bloque determinado, se debe tener presente que la acción de "autonomizar significa ampliar el margen de decisión propia y, normalmente implica por tanto recortar el margen del que disfruta algún otro (...) el logro de una mayor autonomía supone un juego estratégico previo de suma cero, en el cual alguien gana lo que el otro pierde" (Puig, 1984: 44).

El continuo dependencia - autonomía reconoce para el autor antes mencionado la siguiente escala: “1) Dependencia para-colonial; 2) Dependencia nacional; 3) Autonomía Heterodoxa y 4) Autonomía Secesionista” (Puig, 1984: 74).

El secesionismo no es nuevo en política internacional. La secesión significa un desafío global en el cual el país o bloque periférico "corta" el cordón umbilical que lo unía a su metrópoli; como sostiene Puig los "repartidores supremos deciden sin tener en cuenta, espontánea u obligadamente, los intereses estratégicos globales de la potencia dominante como conductora del bloque del cual se retira" (1984: 79).

Es en este sentido que, la propuesta ALBA, como bloque antiimperialista, se encuadraría dentro de la Autonomía Secesionista de Juan Carlos Puig. El fin de la denominada "apertura petrolera" en la Franja del Orinoco, y la salida del Fondo Monetario Internacional y el Banco Mundial serían prueba de este corte.

Al decir de Chávez el pasado día del trabajador en la ciudad española de Barcelona: "Hoy por fin hemos enterrado diez años de apertura petrolera. Desde los pozos hasta el muelle, todo: tanques, carreteras, tuberías, máquinas, sistemas de control y los 4.000 trabajadores que eran contratados por las transnacionales ahora pasan a ser empleados de planta de Petróleos de Venezuela”. Seguidamente en la misma conferencia anunció:

"formalizar la salida de Venezuela del FMI y BM y de todos esos organismos (...) Vamos a salirnos y que nos devuelvan los reales porque nos deben”.

El regreso de "los barriles a sus manos" y lo que ha denominado el presidente "una política sana para la Nación” simbolizarían así un serio corte con los repartidores supremos y el imperialismo, materializado en el poder hegemónico de los Estados Unidos y en las 
instituciones multilaterales de crédito. El presidente Chávez cerró la conferencia del primero de mayo del 2007 con la siguiente afirmación: “El imperialismo dominó nuestra industria básica, nuestros recursos energéticos y nuestros recursos naturales, durante mucho tiempo, eso se acabó hoy. Hoy es el final de esa época donde nuestras riquezas naturales terminaban siempre en manos de cualquiera menos del pueblo venezolano".

De todas formas desde la Teoría, se entiende que la realización de una verdadera propuesta de Autonomía Secesionista sólo es factible en un mundo bipolar, como el de la Guerra Fría; en el cual y según el funcionamiento del sistema internacional, hay un reparto consentido de espacios de influencia entre las superpotencias las que han aceptado cumplir conjuntamente los siguientes criterios: “a) un arsenal de destrucción masiva abrumador respecto del resto de las potencias; b) impermeabilidad inter-bloques; c) autonomización intra-bloques; d) permeabilidad extra-bloques; e) prohibición del uso de la fuerza para pequeños y medianos Estados" (Puig, 1984: 54).

Los Estados al ser gobernados por fuerzas que tengan como objetivo aumentar o afirmar sus márgenes de autonomía respecto de la cabeza del bloque, deben evaluar cuidadosamente y deben conocer en profundidad los datos de la realidad social internacional determinando, con la mayor precisión posible, el margen potencial de decisión autonómica.

El estado estructural del sistema internacional nos indica que, las opciones internacionales son más realizables sobre bases integradas en consonancia con el proceso de globalización actual bajo la hegemonía norteamericana. Como se ha sostenido, "la integración sigue siendo un medio pero: ¿cuál es la vigencia de la autonomía?; y ¿es ésta un medio o un fin en la actualidad? En términos estructurales, la interpretación autonómica sigue estando totalmente vigente, pues en su análisis coincide con la realidad del orden global, con las ecuaciones de poder y por tanto aquí subayace su validez como paradigma analítico, más aún al observar cuán frondosos han sido en los últimos años los trabajos sobre la autonomía periférica latinoamericana" (Oddone, 2004: 107).

Considerando los condicionantes estructurales del sistema internacional actual y tras el fin del sistema bipolar, es que la Autonomía Secesionista no encuentra bases firmes sobre las cuales realizar su proyección sistémica. No obstante ello, la evaluación por exceso de dicho margen es peligrosa; pues genera la reacción del supremo repartidor del bloque 
significando un retroceso difícil de recuperar. Por consiguiente: "la dinámica autonomista suele ser cíclica y desarrollarse por etapas (...) un proceso que consta de avances y retrocesos" (Puig, 1984: 72).

La autonomía sigue siendo un análisis útil para evaluar las ecuaciones de poder, en tanto se condiga con la realidad del sistema internacional en un momento específico. El sistema continúa teniendo un supremo repartidor estructural indiscutible de carácter global, y en este sentido, la secesión no tiene razón de ser en un continente en el cual la potencia hegemónica es hemisférica. Es por ello que, como se verá más adelante, en tanto paradigma analítico sería la Autonomía Relacional la apuesta para estos tiempos.

\section{LA COMUNIDAD SUDAMERICANA DE NACIONES (CSN)}

\section{GÉNESIS DE LA CSN}

El 8 de diciembre de 2004 nació en Cuzco $^{13}$ la propuesta de la CSN, proyecto que pretende incluir a los cinco Estados parte del MERCOSUR: Argentina, Brasil, Paraguay, Uruguay y actualmente Venezuela; a los que se sumaría Chile, los miembros de la Comunidad Andina de Naciones (CAN) entre los que encontramos a Bolivia, Colombia, Ecuador y Perú; y otros dos países del Norte de América del Sur: Guyana y Surinam.

El Producto Bruto Interno (PBI) de la CSN asciende a 973.613 millones de dólares, constituyendo así la quinta potencia mundial; y reúne una población de 361 millones de habitantes, la cuarta al nivel mundial recreando así el espacio para un importante mercado.

Asimismo, la CSN ocupa una superficie superior a los 17 millones de $\mathrm{km}^{2}$; convirtiéndose en la región que más alimentos produce y exporta en el mundo; sus exportaciones suman un total de 181.856 millones de dólares. A su vez, posee el $27 \%$ de agua dulce del mundo; 8 millones de $\mathrm{km}^{2}$ de bosques; 2 océanos que bañan sus costas y una reserva de hidrocarburos a cien años.

Otro de los factores que confluye a favor de esta propuesta de integración es que todos los países de la región tienen una historia común y valores compartidos; el $95 \%$ de

\footnotetext{
${ }^{13}$ La palabra Cuzco (también aceptada como Cusco) procede de una voz quechua que significa "ombligo del mundo", convirtiéndose así en la sede adecuada por un doble simbolismo: la independencia de los pueblos sudamericanos hace ciento ochenta años ("nacimiento") y la nueva independencia sudamericana del siglo XXI (el "renacimiento" del pueblo sudamericano en un todo regional).
} 
sus habitantes tiene una misma religión y se caracteriza por dos lenguas mutuamente inteligibles.

"Cusco es digna casa para este logro. Fue capital del Incario, y el Incario fue el primer proyecto de unión de nuestra región, abortado por la Conquista cuando avanzaba en la ligazón de otros pueblos originarios con el sistema que se había articulado a lo largo de los Andes. La conquista y la colonización ibéricas fueron, a su dolorosa manera, otras formas de mantener unida a América del Sur aún bajo la dependencia de las metrópolis peninsulares. No evitó, sin embargo, que cuando nuestros pueblos se sintieron maduros para la vida independiente, la consagraran a sangre y fuego. El sueño emancipador de gigantes como Bolívar, San Martín, O'Higgins, Sucre y tantos otros parecía imposible en el comienzo y, pese a todo, probamos que podía ser vivido también en la vigilia" (Bielsa, 2004).

Desde la perspectiva de los propios líderes que asistieron al encuentro en Perú, constituiría el "renacimiento de una región" en el sentido de responder al imperativo histórico y presente de los libertadores de estas tierras como Simón Bolívar, José de San Martín, Bernardo O'Higgins, Antonio Sucre: la realización del "sueño bolivariano".

Si bien en la construcción del escenario sudamericano no priman las estructuras favorables, todo proceso de integración es un proceso humano, y en cuanto tal el mismo tomará el rumbo y movimiento que le den los hombres interesados y participantes del mismo. La voluntad política sostenida de estos hombres puede llegar a ser la base estructurante de la nueva realidad.

\section{LA DECLARACIÓN DE CUZCO Y SUS LÍNEAS DE ACCIÓN}

En el marco de los actos conmemorativos del ciento ochenta aniversario de las batallas de Junín y Ayacucho que sellaron la independencia de América del Sur se realizó el III Encuentro de Presidentes de dicha subregión.

La Declaración de Cuzco establece en el punto II, primer párrafo, que el espacio sudamericano integrado se desarrollará y perfeccionará impulsando "la concertación y 
coordinación política y diplomática que afirme a la región como un factor diferenciado y dinámico en sus relaciones externas".

Autores sudamericanos como Juan Carlos Puig y Helio Jaguaribe han juzgado la concertación política y la integración económico regional o subregional como condiciones necesarias para el logro de una mayor autonomía. Concertación e integración, en un primer momento, no se percibieron como necesariamente autonomizantes, sino como instrumentales al proceso de construcción y preservación de la autonomía. Como sostuviera Juan Carlos Puig (1980: 155): "tal vez porque los objetivos no fueron propiamente autonómicos es que no han avanzado decididamente los procesos de integración en América Latina".

En el párrafo segundo del mencionado punto, la Declaración insta a "la profundización de la convergencia entre el MERCOSUR, la Comunidad Andina y Chile a través del perfeccionamiento de la zona de libre comercio, apoyándose en lo pertinente en la Resolución 59 del XIII Consejo de Ministros de la ALADI del 18 de octubre de 2004, y su evolución a fases superiores de la integración económica, social e institucional. Los Gobiernos de Surinam y Guyana se asociarán a este proceso, sin perjuicio de sus obligaciones bajo el Tratado revisado de Chaguaramas".

El establecimiento de un TLC sobre bases mutuamente consensuadas y que permita recrear escenarios equilibrados de intercambio comercial constituirá el punto de partida para la evolución a fases superiores, no sólo de integración económica, sino también social e institucional.

Asimismo, el párrafo tercero establece que deberá impulsarse "la integración física, energética y de comunicaciones en Sudamérica sobre la base de la profundización de las experiencias bilaterales, regionales y subregionales existentes, con la consideración de mecanismos financieros innovadores y las propuestas sectoriales en curso que permitan una mejor realización de inversiones en infraestructura física para la región”.

Lo antes mencionado se intentará concretar en el marco de la Iniciativa para la Integración de la Infraestructura Regional Sudamericana (IIRSA). Esta iniciativa es un programa que incluye a los doce países de América del Sur y que se originó en la I Reunión de Presidentes de América del Sur realizada en Brasilia en el año 2000. 
El IIRSA constituye la consolidación del compromiso de los gobiernos sudamericanos con la modernización e integración de la infraestructura regional sobre la base de tres agencias multilaterales, como la Corporación Andina de Fomento (CAF), el Banco Interamericano de Desarrollo (BID) y el Fondo Financiero para el Desarrollo de la Cuenca del Plata (FONPLATA); y en su fase de ejecución se pretende adoptar la modalidad de las denominadas Parcerías Público Privadas o Public Private Partnership (PPP) en el desarrollo del concepto de integración regional descentralizada.

Las vías de acción escogidas para el desarrollo de la infraestructura ponen de relieve la importancia de la misma para el incremento de los intercambios comerciales y el aumento de la competitividad regional, involucrándose en una visión geoeconómica del desarrollo.

La Declaración en el párrafo cuarto establece como necesaria "la armonización de políticas que promuevan el desarrollo rural y agroalimentario". Sabido es que los países sudamericanos, y los del MERCOSUR en particular, son grandes productores de ciertos bienes agrícola ganaderos que están en condiciones de colocar en terceros mercados a precios sumamente competitivos; pero que por motivos de una suerte de competencia desleal plasmada en los subsidios norteamericanos y europeos al agro no logran encontrar su espacio en los mercados internacionales.

En el párrafo quinto, la Declaración sostiene que deberá impulsarse "la transferencia de tecnología y de cooperación horizontal en todos los ámbitos de la ciencia, educación y cultura". El cambio tecnológico se introduce aquí como un nuevo concepto que merece ser analizado, tomando como premisa que solo logran desarrollarse en este mundo globalizado aquellos países que participan de la globalización a partir de procesos autocentrados, el "cambio tecnológico endógeno" se presenta como un factor favorecedor del desarrollo autocentrado en el contexto global y a escala regional.

En el último párrafo del punto II de la Declaración se considera fundamental "la creciente interacción entre las empresas y la sociedad civil en la dinámica de integración de este espacio sudamericano, teniendo en cuenta la responsabilidad social empresarial". La necesidad de una nueva clase empresaria, estructurada sobre los cimientos de una relación empresa - sociedad civil de mutuo beneficio, es fundamental para el crecimiento simétrico a escala regional. 


\section{LOS PAÍSES DE LA SUBREGIÓN FRENTE A LA CSN}

Es en este marco que debemos analizar caso por caso y brevemente la expresión de la voluntad política en los distintos países y los posibles beneficios y presentes intereses en esta nueva propuesta de integración.

La voluntad política expresada por los presidentes sudamericanos es dispar. En primer lugar encontramos a Luiz Inácio Lula da Silva de Brasil, quien logra finalmente desarrollar la vieja idea del ALCSA (Área de Libre Comercio de Sudamérica), propuesta originariamente por el gobierno de Itamar Franco en 1993 (Moniz Bandeira, 2004: 504) y retomada por su antecesor Fernando H. Cardoso ${ }^{14}$.

El ALCSA es el antecedente inmediato de la CSN, recreándose así la vieja idea de la Unión de los Países del Sur ${ }^{15}$ bajo el liderazgo brasileño. No podríamos entender hoy día el proceso sudamericano sin la presencia y el rol activo de Brasil, quien cuenta con el $72 \%$ de la superficie y el $80 \%$, tanto de la población como del PBI del MERCOSUR.

Este liderazgo que reclama el vecino país se debe tanto a su peso específico como al estancamiento económico e institucional de su contrapeso histórico en la región desde inicios del siglo XIX, la Argentina. A esta cuestión propia de la relación bilateral debe sumarse la óptica con que Brasil mira y se ve a sí mismo en el escenario internacional ${ }^{16}$.

\footnotetext{
${ }^{14}$ La propuesta ALCSA resurgió en la I Cumbre de Presidentes de América del Sur de Brasilia del año 2000 dejando en claro, al valorizar el concepto de América del Sur en lugar del de América Latina; que había en danza dos proyectos paralelos e independientes para el continente americano. En aquella oportunidad se definió como objetivo primordial la realización de un tratado de libre comercio entre el MERCOSUR y la CAN que constituyese "la espina dorsal de América del Sur". Se pretendía así fortalecer la capacidad negociadora de América del Sur particularmente frente a los Estados Unidos y su propuesta ALCA.

${ }^{15}$ La Unión de los Países del Sur recrea el ideario integracionista del presidente argentino Juan Domingo Perón sobre la base y reformulación de los acuerdos $\mathrm{ABC}$ del brasileño Río Branco. "Como corolario de los esfuerzos de integración subregional se desarrolló entre 1946 - 1948 el primer ciclo de integración, con acuerdos bilaterales preferenciales o de unión aduanera, en particular con los países limítrofes. Tal estrategia surgió como camino factible para superar recelos, desconfianzas, conflictos pendientes, y así acceder paso a paso a una unión económica generalizada en el Cono Sur" (Oddone, 2004: 11).

16 "Brasil interpreta que las relaciones internacionales se basan en presupuestos realistas, el sistema internacional es concebido de manera jerárquica y oligopólica, concibe los regímenes e instituciones internacionales primariamente como reflejo de la distribución estructural de poder ajustando su accionar en ellos a través del cálculo costo- beneficio, haciendo que la participación en los mismos sea fruto de decisiones racionales y no idealistas. Sennes caracteriza la política exterior brasileña por una fuerte adhesión a los principales regímenes internacionales, una clara regionalización a las principales iniciativas del país y por la adopción de estrategias basadas en el reconocimiento de la existencia del conflicto Norte-Sur" (Oddone y Granato, 2004: 13).
} 
En todo proceso de integración siempre hay líderes, comúnmente denominados "locomotoras" de la integración. Para este caso se necesita entonces de un liderazgo consentido y consensuado (Russell y Tokatlián, 2003).

Brasil es seguido de cerca por Venezuela, a la búsqueda de concretar su idea histórica de una América del Sur unida. Más allá de esta determinación histórica, la CSN es para Venezuela un proyecto esencial desde su perspectiva de acercamiento con Brasil. "Desde hace más de una década Venezuela se aproxima a Brasil, por su complementariedad industrial y energética, por su propósito de desarrollar las regiones del sur y el oriente del país y como mecanismo de equilibrio diplomático multilateral frente a Estados Unidos" (Cardona, 2005).

Ésta última es continuada por Colombia. El caso de éste país adquiere determinadas singularidades. Debemos tomar en cuenta su fuerte pertenencia en la CAN y la importancia que tiene para el propio país un buen nivel de relación con sus vecinos; pues esto le permite aumentar su nivel de autonomía frente a los Estados Unidos; con quien ha negociado un TLC pendiente de ratificación y a quien está unido a través del Plan Colombia.

Mucho dependerá de la actitud y de la aptitud de los vecinos países como Brasil, Venezuela y Perú para fortalecer la posibilidad de acercar a Colombia a la CSN y en este marco de concertación poder responder a los problemas internos que la aquejan.

Ya desde la presidencia de Ricardo Lagos, se observaba en Chile una relativa voluntad política que se entendía en el marco de una política comercial exterior distinta a la del resto de los demás países de la subregión; caracterizada por su posición de global trader, apertura económica unilateral y regionalismo abierto. Como ha afirmado la presidente Michelle Bachelet, en términos geoestratégicos; la CSN adquiere importancia para Chile a los efectos de satisfacer su provisión de materias primas, energía, mercados y ampliación de su área de influencia multilateral siempre que ello implique la puesta en marcha de un TLC entre los países sudamericanos.

En el caso de Bolivia, la CSN se presenta como el espacio propicio para que el país se convierta en la "bisagra regional" entre la CAN y el MERCOSUR. Debemos recordar así que Bolivia constituyó el primer país asociado del MERCOSUR, ya habiendo hecho explícita su voluntad de ingreso como Estado parte en el corto plazo. La CSN como espacio 
de concertación y coordinación política puede ofrecer a Bolivia escenarios crecientes de estabilidad para su situación en términos de gobernabilidad política.

Asimismo, la CSN podría contribuir al saldo de la deuda histórica de Chile y Perú con Bolivia por la falta de una salida al mar, garantizándole en dicho marco de concertación subregional puertos con salida al océano Pacífico.

En el caso ecuatoriano, la baja voluntad política demostrada inicialmente por el ex presidente Lucio Gutiérrez ponía de manifiesto la importancia de la relación bilateral Ecuador - Estados Unidos y la dolarización de la economía nacional. Pero tampoco Ecuador debiera estar completamente ausente, pues por medio de la CSN podría recuperar algún grado de acceso significativo a la cuenca amazónica que siempre le fue disputado y vedado por Perú. Con el cambio de gobierno, y la asunción de Rafael Correa Delgado es de esperar que el involucramiento del Ecuador con la CSN crezca a partir de los puntos en común y cosmovisión política compartida con los restantes presidentes de Sudamérica.

Por su parte, Paraguay, Uruguay y Argentina han demostrado un bajo interés en este nuevo proyecto integracionista. "Así, mientras en Cusco [de 2004] estaban ausentes Jorge Batlle, Néstor Kirchner y Nicanor Duarte Frutos (...) es la representación plástica de una fragmentación predominante entre los gobiernos que, al mismo tiempo, concurren a un proyecto unificador" (Bilbao, 2004).

Una incorporación total de los países de Sudamérica redundaría en un aumento de las inversiones recíprocas y de las ventajas geopolíticas, estructuradas a partir de sistemas administrativos conjuntos. La capacidad de estabilización sobre los vecinos en problemas y el incremento general y sostenido del potencial colectivo en las negociaciones internacionales, generando vis-à-vis el aumento relativo de la capacidad autonómica subregional.

\section{UNA AUTONOMÍA REDEFINIDA PARA LA PROPUESTA CSN}

Como reza la Declaración de Cuzco en sus primeros párrafos: "La historia compartida y solidaria de nuestras Naciones, que desde la gesta de la Independencia han enfrentado desafíos internos y externos comunes, demuestra que nuestros países poseen potencialidades aún no aprovechadas tanto para utilizar mejor sus aptitudes regionales como para fortalecer las capacidades de negociación y proyección internacionales". 
A continuación se sostiene: "El pensamiento político y filosófico nacido de su tradición, que reconociendo la preeminencia del ser humano, de su dignidad y derechos, la pluralidad de pueblos y culturas, ha consolidado una identidad sudamericana compartida y valores comunes, tales como: la democracia, la solidaridad, los derechos humanos, la libertad, la justicia social, el respeto a la integridad territorial, a la diversidad, la no discriminación y la afirmación de su autonomía, la igualdad soberana de los Estados y la solución pacífica de controversias".

Tomando en consideración lo hasta aquí expuesto es que retomamos la idea de afirmación de la autonomía, entendiendo a esta última no como un derecho de los Estados; sino como una propiedad cambiante y un propósito básico de toda política exterior.

La autonomía ha sido por tanto, una preocupación histórica de las políticas exteriores de los países de América del Sur ${ }^{17}$; que en el estado actual de la dinámica de la integración muta su base nacional por una regional a los efectos de aumentar dichos márgenes de autonomía para la toma de decisiones en un mundo global.

Las circunstancias mundiales de globalización y fin de la Guerra Fría que encontraron su justificación y razón de ser a lo largo de la década del noventa y las circunstancias nacionales - regionales de democratización e integración en el Cono Sur han modificado lo que un autor ha denominado "marco para la acción" (Cox, 1986); presentándose así la necesidad de una resignificación profunda del concepto de autonomía.

Es este nuevo contexto el que favorece el tránsito de una autonomía que se definía por contraste a otra que se construye en el marco relacional. La Autonomía Relacional debe entenderse como la capacidad y disposición de un Estado para tomar decisiones con otros por propia voluntad y para hacer frente en forma conjunta a situaciones y procesos ocurridos dentro y fuera de sus fronteras. La Autonomía Relacional implica por tanto, la capacidad y disposición del "nosotros" y del "nosotros - otros".

\footnotetext{
${ }^{17}$ Como nos recuerdan Russell y Tokatlián (2002: 168) “el tema de la autonomía fue un asunto más sudamericano que latinoamericano. En la América Latina del Norte (de la que forman parte México, Centroamérica y el Caribe), el acento estuvo puesto más en la soberanía 'legal internacional' y 'westfaliana vatteliana', debido a que esta región históricamente fue objeto de diversas acciones coercitivas y de fuerza por parte de Washington (...). Por su parte, América del Sur, desde Colombia hasta la Argentina, dispuso de un margen de maniobra diplomático, comercial y cultural relativamente mayor frente a Washington. No es sorprendente entonces, que la literatura principal sobre el tema se haya producido en América del Sur y, más específicamente, en el Cono Sur".
} 
La manifiesta complejidad del sistema mundial actual está dada por la aparición de problemáticas sin precedentes, que supera la escala de gobernabilidad de los Estados Nación y sus limitadas dinámicas y formas de articulación de políticas conjuntas (Zacher, 1992). En este mismo sentido, la significativa des-territorialización de la dinámica productiva capitalista, cuyos flujos de inversión, producción y consumo han derribado las fronteras estatales, culturales e ideológicas, sometiendo a un profundo replanteo la existencia del Estado como escala moderna y eficaz de gobernabilidad.

Esta nueva concepción de la autonomía implica, en principio, una estrategia de regionalización que involucra una entrega voluntaria y creciente de soberanía que debe traducirse en la creación de instituciones que apuntan como base a la noción del bien común, eje central de la gobernabilidad.

La Autonomía Relacional requiere de una confianza firme, de una predisposición sin límite y de una capacidad de trabajo sostenida en aras del bien común. Estas claves de la integración regional, y la política exterior consecuente que se debe desarrollar con y en el marco de la CSN, cobraran sólo sentido si se reconocen los intereses fundados de las partes del pueblo sudamericano y se abandonándose las poses vanas de nuestros líderes pues la CSN durará tanto "como buenos sean los hombres que representen la dirección".

\section{CONCLUSIÓN}

Analizar las nuevas propuestas de integración al nivel subsistémico reviste siempre importancia a los efectos de reconocerlas como intentos autonómicos sobre la base regional. En este sentido se debe tener siempre en claro: el qué, el por qué y el para qué integrarse. El ALBA y la CSN reconstruyen este completo horizonte integrativo.

Más allá de las propias posibilidades de mantenerse en el tiempo; y de los apoyos y contras que las mismas suscitan, ambas implican per se un planteo autonómico diferenciado. En cuanto tal, se entiende que por el tipo de Autonomía Relacional que conlleva la CSN, las posibilidades de su concreción en la actual etapa de globalización parecen ser mayores.

Los planteos de autonomía secesionista simbolizan otra época del sistema internacional, que si bien ya era en cierta forma globalizado, la estructura de poder del mismo era bipolar y constituía un juego de suma cero, por tanto la autonomía se construía 
en ese espacio intra-bloque. La falta de bloques políticos altamente identificables ha llevado a definir de una forma nueva la autonomía, transformándose en una autonomía relacional, en la cual los enfrentamientos entre "el yo y los otros" pasan a ser parte del "nosotros-otros" en un juego de suma positiva sobre las características del regionalismo abierto.

\section{REFERENCIAS BIBLIOGRÁFICAS}

- BIELSA, Rafael. "Nuestro destino sudamericano", publicado en Diario Clarín, [http:/www.clarin.com/diario/2004/12/08/opinion/o-883234.htm]. Disponible: 12/08/2004. - BILBAO, Luis. "Comunidad Suramericana de Naciones. Anuncios de una nueva era en Cusco y Ayacucho", publicado en Le Monde Diplomatique, edición Cono Sur, [http://www.eldiplo.org]. Disponible: 12/04/2005.

- BOISIER, Sergio. “Hay espacio para el Desarrollo Local en la Globalización?”. Revista de la CEPAL, Nro. 86, 2005.

- CARDONA, Diego. “¿Tiene futuro la Comunidad Sudamericana de Naciones?”, publicado en Foreign Affairs en Español, [http://www.foreignaffairs-esp.org/20050401faen espessay050209/diego-cardona-c/tiene-futuro-la-comunidad-sudamericana-denaciones.htm 1]. Disponible: 01/04/2005.

- COX, Robert. Social Forces, State and World Orders: beyond International Relations Theory. En KEOHANE, Robert (Ed.). Neorrealism and its Critics. New York: Columbia University, 1986.

- Discurso en la Clausura de la III Cumbre de los Pueblos de América. Estado Mundialista de Mar del Plata, Argentina; 4 de noviembre de 2005. Accesible desde: [http:/www.altern ativabolivariana.org/pdf/discurso_Chavez_MardelPlata.pdf]. Disponible: 19/02/2007.

- KATZ, Claudio. "Las disyuntivas del ALBA", publicado en Rebelión, [http://www.rebelion.org/docs/29179.pdf.htm]. Disponible: 20/03/2007.

- MARTÍNEZ, Benjamín. “Cosmovisión, ambiente y desarrollismo periférico: nuevas tendencias glocales en el ambientalismo contemporáneo". En: Actas del II Simposio Ambiente y Desarrollo, Facultad de Ingeniería, Universidad Central de Venezuela, 2004. 
- MARTÍNEZ, Benjamín. "El ALBA desde la Globalización”, publicado en Encontrarte, [http://encontrarte.aporrea.org/teoria/sociedad/30/a9607.htm]. Disponible: 25/02/2007.

- MONIZ BANDEIRA, Luiz A. Argentina, Brasil y Estados Unidos. De la Triple Alianza al MERCOSUR. Buenos Aires: Grupo Editorial Norma, 2004.

- MONTERO SOLER, Alberto. "ALBA: avances y tensiones en el proceso de integración popular bolivariano". Revista Ágora, Vol. III, Nro. 15, 2007.

- ODDONE, Carlos Nahuel. El Primer Ciclo de la Unión de los Países del Sur en las propuestas de integración del Peronismo. Tesis de Maestría en Derecho de la Integración Económica, Facultad de Ciencias Jurídicas de la Universidad del Salvador en convenio con l'Université Paris 1 Panthéon-Sorbonne. Buenos Aires - Paris, 2004.

- ODDONE, Carlos Nahuel y GRANATO, Leonardo. "México en un eventual acuerdo de libre comercio de las Américas: perspectivas y aproximación desde la historia actual". Revista Tendencias, Vol. VII, Nro. 1, 2006.

- ODDONE, Carlos Nahuel y GRANATO, Leonardo. "La política exterior de Brasil frente al MERCOSUR", publicado en Diario El Eco de Tandil, 19/12/2004.

- ODDONE, Carlos Nahuel y GRANATO, Leonardo. "El peronismo y la búsqueda de la autonomía heterodoxa: La Tercera Posición en política exterior". En: Actas del I Encuentro del CERPI "Veinte años de política exterior en democracia". Centro de Reflexión en Política Internacional del Instituto de Relaciones Internacionales, Universidad de La Plata. La Plata, 30 de octubre de 2003.

- PUIG, Juan Carlos. Doctrinas internacionales y autonomía latinoamericana. Caracas: Universidad Simón Bolívar, 1980.

- PUIG, Juan Carlos (Comp.). América Latina: Políticas Exteriores Comparadas, Tomos I y II. Buenos Aires: Grupo Editor Latinoamericano, 1984.

- RUSSELL, Roberto y TOKATLIÁN, Juan Gabriel. "De la autonomía antagónica a la autonomía relacional: una mirada teórica desde el Cono Sur". Revista Latinoamericanos, Nro. 21, 2002.

- RUSSELL, Roberto y TOKATLIÁN, Juan Gabriel. El lugar de Brasil en la política exterior argentina. Buenos Aires: Fondo de Cultura Económica, 2003.

- VERTBISKY, Horacio. "Tres modelos", publicado en Diario Página/12, [http://www.pagina12.com.ar/diario/elpais/1-81553.html]. Disponible: 11/03/2007. 
- ZACHER, Mark (1992). The Decaying Pillars of the Westphalian Temple: implications for international order and governance. En: ROSENAU, James y CZEMPIEL, Ernest Otto (Eds.). Governance without government. Cambridge: Cambridge University Press, 1992. 